\title{
Proteomics and microstructure profiling of goat milk protein after homogenization
}

\author{
D. Chen, ${ }^{1}$ X. Y. Li, ${ }^{1}$ X. Zhao, ${ }^{1}$ Y. S. Qin, ${ }^{1}$ X. X. Zhang, ${ }^{1}$ J. Li, ${ }^{1}$ J. M. Wang, ${ }^{2}$ and C. F. Wang ${ }^{1 *}$ \\ ${ }^{1}$ College of Food Science and Engineering, Qilu University of Technology (Shandong Academy of Sciences), Jinan, China 250353 \\ ${ }^{2}$ College of Animal Science and Veterinary Medicine, Shandong Agricultural University, Taian, China 271018
}

\begin{abstract}
This study investigated the protein changes in goat milk during the homogenization process using label-free quantification. We quantified 310 and 315 proteins in the control group (CG) and homogenized group (HG), respectively, and 16 proteins were significantly different between the 2 groups. For HG, the goat milk protein particle sizes were smaller and more evenly distributed and exhibited an increase in the regular arrangement of the secondary structures. Proteomics analysis verified that xanthine dehydrogenase and asparaginase-like 1 expression in CG were higher than in HG, whereas the opposite was observed for fructose-bisphosphate aldolase, $\kappa$-casein, and $\beta$-casein. Significant changes were found in the homogenization-treated goat milk proteome that were related to goat milk glycolysis/gluconeogenesis metabolism. This work provides updated information on the current proteome characteristics of homogenized goat milk, which may be important for applying the protein component of goat milk to human nutrition and health.
\end{abstract}

Key words: goat milk, homogenization, proteomics, microstructure

\section{INTRODUCTION}

Homogenization has become a standardized processing method in the dairy industry. In homogenization, mechanical forces reduce fat globule size, alter the structure of milk proteins, and reduce enzyme activity and vitamin levels (Pereda et al., 2008). Temperature elevation also occurs due to a variety of phenomena, including shear stress, turbulence, cavitation, and the transformation of kinetic energy into heat during the pressure increase (Amador-Espejo et al., 2014). These modifications generate a synergistic effect with the

Received July 11, 2018.

Accepted January 7, 2019.

*Corresponding author: cunfangwang@163.com mechanical changes happening during the homogenization process. Although homogenization reduces Trp fluorescence intensity, it does not significantly change the tertiary or secondary structure of casein (Qi et al., 2015).

In recent years, the relatively new and rapidly developing field of proteomics has offered a key technique to identify, characterize, and quantify proteins. Goat milk proteins are composed of caseins $(\alpha-, \beta-$, and $\kappa-\mathrm{CN})$, whey proteins (WP; $\alpha-\mathrm{LA}, \beta-\mathrm{LG}$, BSA, immunoglobulins, and other minor proteins), proteose peptones, and membrane proteins [mostly milk fat globule membrane protein (MFGMP); Chen et al., 2018; Li et al., 2018a]. Furthermore, $9 \alpha_{\mathrm{S}^{-}} \mathrm{CN}, 4 \alpha_{\mathrm{S}^{2}} \mathrm{CN}, 12 \beta-\mathrm{CN}, 14 \kappa-\mathrm{CN}$, $3 \alpha$-LA, and $11 \beta$-LG have been identified in bovine milk protein (Le et al., 2017). A variety of milk proteins have been verified by applying quantitative proteomics and bioinformatics to milk proteins using label-free quantification (Anagnostopoulos et al., 2016; Lu et al., 2016).

During conventional homogenization, finer emulsions are achieved from smaller fat globules, improving the digestibility and absorption of milk fat (Brick et al., 2017). The natural fat globules (diameter $1-10 \mu \mathrm{m}$ ) are broken into small globules (diameter $<1 \mu \mathrm{m}$ ), resulting in the destruction of MFGMP (Qi et al., 2015) and inadequate coverage of the newly formed fat surface, even if a portion of native membrane remains associated with the fat droplets (Huppertz, 2011). In addition, homogenization to reduce the size of the fat globules also substantially changes casein, WP, and MFGMP by altering protein-protein interactions. To cover the increased fat globule surface area and prevent the fat from coalescing in homogenized milk, surface-active proteins, especially casein, are adsorbed onto newly formed globules. Treated milk is whiter than raw milk due to the increase in the number of fat globules, which diffract light more efficiently. Caseins are preferentially adsorbed over WP at the oil-water interface in homogenized milk (Cano-Ruiz and Richter, 1997), which could be useful for fine-tuning processing conditions when manufacturing dairy products (Liang et al., 2017). 


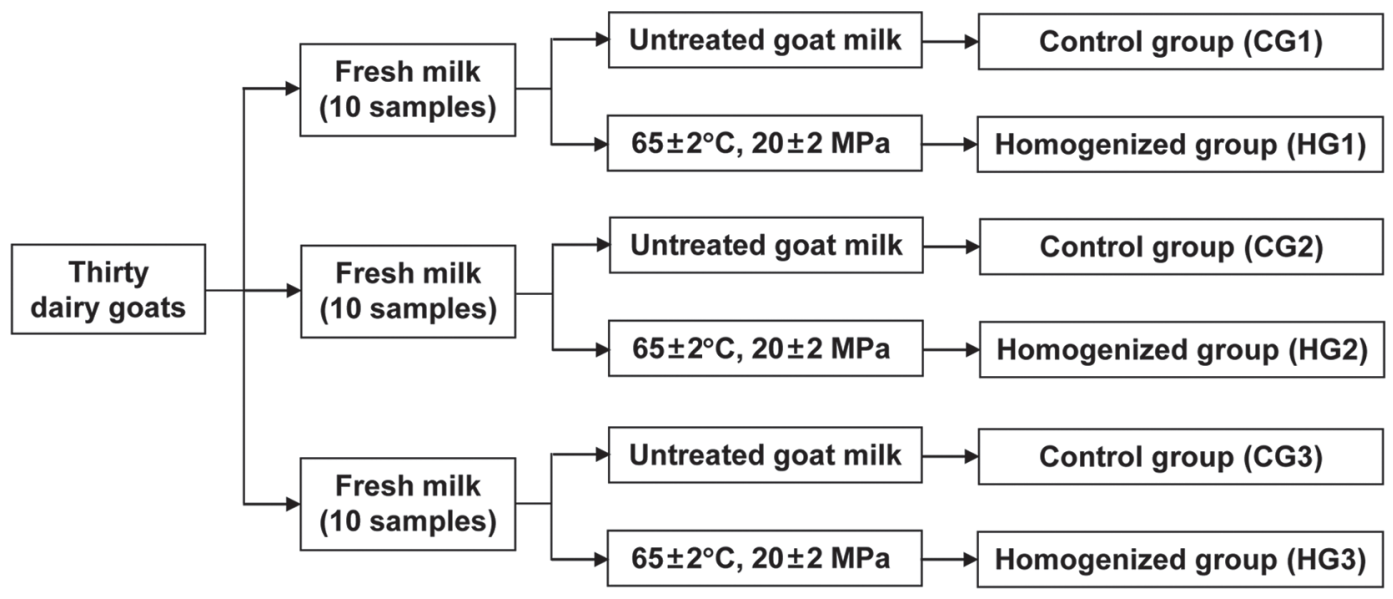

Figure 1. Workflow for collection and preparation of control and homogenized goat milk samples.

Previous studies focused on the effects of temperature, pressure, time, and number of treatments on fats, proteins, and the activity of enzymes and vitamins in dairy products (Pinho et al., 2011a). Changes in protein functionality as well as protein and fat absorption after homogenization have also been assessed (Ye et al., 2017); however, no one has studied the proteome of goat milk after homogenization. In the present study, the changes in goat milk protein composition and expression during homogenization processing were identified via label-free technology based on the MaxQuant algorithm for fast and cost-efficient protein quantification. Gene Ontology (GO; http://www.geneontology.org/) annotation and the Kyoto Encyclopedia of Genes and Genomes (KEGG; https://www.genome.jp/kegg/) were used to analyze and classify proteomics data, and morphological analysis of goat milk protein was used to predict microstructural changes in the protein in homogenized goat milk. The effect of homogenization on the glycolytic pathway was then evaluated by comparing changes in homogenized milk protein expression. This work expands our knowledge of the goat milk proteome and is helpful to further clarify the differences between homogenized and fresh goat milk.

\section{MATERIALS AND METHODS}

\section{Sample Collection and Preparation}

Thirty Laoshan goats aged 30 to 32 mo with no signs of acute mastitis or other clinical diseases were selected from Three Hi Goat Farms in Shandong Province (Zhujiayu Village, Daolang Town, Tai'an City, China). This farm is well organized and uses freestall housing and a TMR silage diet. Feces are cleared manually and milking is performed manually at $1000 \mathrm{~h}$. All goat milk samples were collected at $75 \mathrm{~d}$ after lactation and transported to the laboratory with dry ice within 1 h. Fresh mature milk samples from these goats were randomly divided into 3 groups, with 10 in each group, and pooled. These 3 mixed mature milk samples were filtered and divided into 2 groups again for a total of 6 samples. The samples in the first group served as the control group $(\mathbf{C G})$ and the other group was the homogenized group (HG), which was treated at $65 \pm$ $2^{\circ} \mathrm{C}$ and $20 \pm 2 \mathrm{MPa}$ (Figure 1). Whole-milk samples were supplemented with SDT lysis buffer (4\% SDS, 100 $\mathrm{mmol} / \mathrm{L}$ Tris- $\mathrm{HCl}, 1 \mathrm{mmol} / \mathrm{L}$ dithiothreitol, $\mathrm{pH}$ 7.6) and boiled for 15 min. After centrifugation at 14,000 $\times g$ for $40 \mathrm{~min}$ at $4^{\circ} \mathrm{C}$ (Eppendorf 5430R, Hamburg, Germany), the content of protein in the aqueous phase was determined using the BCA Protein Assay Kit (Bio-Rad, Hercules, CA). The supernatant was used for filter-aided sample preparation as described below (Wisniewski et al., 2009).

\section{Filter-Aided Sample Preparation}

Protein samples $(200 \mu \mathrm{g})$ were diluted in SDT buffer, incubated for $5 \mathrm{~min}$ in boiling water, and cooled to room temperature. Two centrifugations at 14,000 $\times g$ for 15 min at $4^{\circ} \mathrm{C}$ were performed after adding 2 volumes of $200 \mu \mathrm{L}$ of UA buffer $(8 \mathrm{~mol} / \mathrm{L}$ urea, 150 $\mathrm{mmol} / \mathrm{L}$ Tris-HCl, $\mathrm{pH}$ 8.0). We then added $100 \mu \mathrm{L}$ of $100 \mathrm{mmol} / \mathrm{L}$ iodoacetamide (163-2109, Bio-Rad) in UA buffer and the samples were incubated in the dark for $30 \mathrm{~min}$. The filters were washed 3 times with 100 $\mu \mathrm{L}$ of UA buffer and twice with $100 \mu \mathrm{L}$ of $25 \mathrm{mmol} / \mathrm{L}$ $\mathrm{NH}_{4} \mathrm{HCO}_{3}$ buffer. Trypsin buffer $(4 \mu \mathrm{g}$ of trypsin in $40 \mu \mathrm{L}$ of $100 \mathrm{mmol} / \mathrm{L} \mathrm{NH}_{4} \mathrm{HCO}_{3}$ ) was then added to 
Table 1. Description of related parameters for data analysis

\begin{tabular}{ll}
\hline Item & Value \\
\hline Enzyme & Trypsin \\
Maximum missed cleavages & 2 \\
Main search & $6 \mathrm{ppm}$ \\
First search & $20 \mathrm{ppm}$ \\
MS/MS tolerance & $20 \mathrm{ppm}$ \\
Fixed modifications & Carbamidomethyl (C) (Pxidation (M), acetyl (protein N-term) \\
Variable modifications & Oniprot_Caprinae_32203_20170613.fasta \\
Database & Reverse \\
Database pattern & $\leq 0.01$ \\
Peptide false discovery rate (FDR) & $\leq 0.01$ \\
Protein FDR & 2 min \\
Time window (match between runs) & Razor and unique peptides were used for protein \\
Protein quantification & quantification \\
& True \\
Label-free quantification (LFQ) & 1 \\
LFQ minimum ratio count & \\
\hline
\end{tabular}

the samples overnight at $37^{\circ} \mathrm{C}$. The peptides from each sample were desalted on $\mathrm{C} 18$ cartridges [Empore C18 SPE cartridges (standard density); bed i.d., $7 \mathrm{~mm}$; volume, $3 \mathrm{~mL}$; Sigma-Aldrich, St. Louis, MO] and reconstituted in $40 \mu \mathrm{L}$ of $0.1 \%$ ( $\mathrm{vol} / \mathrm{vol}$ ) formic acid (Wisniewski et al., 2009). These samples were prepared for analysis using LC/MS.

\section{LC/MS}

The peptide mixture was injected onto a reverse-phase trap column (Acclaim PepMap100, $100 \mu \mathrm{m} \times 2 \mathrm{~cm}$, nanoViper C18, Thermo Fisher Scientific, Waltham, MA) followed by a C18 reverse-phase analytical column (Easy Column, $10 \mathrm{~cm}$ long, $75 \mu \mathrm{m}$ inner diameter, $3 \mu \mathrm{m}$ resin, Thermo Fisher Scientific). The mobile LC phase contained buffer A ( $0.1 \%$ formic acid) and B ( $84 \%$ acetonitrile and $0.1 \%$ formic acid) and was eluted at a flow rate of $300 \mathrm{~nL} / \mathrm{min}$ with linear gradients. The MS was programmed using a data-dependent top-10 method. The survey scan was from 300 to $1,800 \mathrm{~m} / z$ with a resolution of 70,000 at $200 \mathrm{~m} / z$ (Zhu et al., 2014).

\section{ELISA}

Two candidate proteins ( $\kappa-\mathrm{CN}$ and $\beta-\mathrm{LG}$ ) were chosen to further verify the proteomics results because they are abundant and play an important role after homogenization. The content of $\kappa-C N$ (http://www .jonln.com/) and $\beta$-LG (http://www.cloud-clone.com/) in different samples was measured according to the manual provided with the ELISA kits. The ELISA kit for $\kappa$-CN (cat. no. JL46016) was obtained from Jianglai Biological (Shanghai, China); the ELISA kit for $\beta$-LG (cat. no. SEBO23 Bo) was obtained from Cloud-Clone Corp. (Wuhan, China).

\section{Data Analysis}

The MS data were analyzed using MaxQuant software version 1.3.0.5 (Max Planck Institute of Biochemistry, Martinsried, Germany) with the parameters in Table 1 (Cox et al., 2014). Contaminated protein was discarded during this process.

\section{Cluster Analysis, GO, and KEGG Enrichment Analyses}

Protein expression data were used to perform hierarchical clustering analysis. Cluster 3.0 (http://bonsai .hgc.jp/ mdehoon/software/cluster/software.htm) and Java Treeview software (http://jtreeview.sourceforge .net) were used. The goat reference database for differentially expressed protein searches was downloaded as FASTA files from the UniProtKB database (http:/ /www.uniprot.org/, June 13, 2017, release; Götz et al., 2008). The FASTA protein sequences of differentially expressed proteins were compared with online GO database to retrieve GO and were subsequently mapped to pathways in KEGG UniProt-Caprinae database (Moriya et al., 2007).

\section{Field Emission Scanning Electron Microscope}

A 10\% acetic acid solution was used to adjust the $\mathrm{pH}$ (4.1) to the casein isoelectric point for casein and WP collection (Zhu et al., 2018). The WP and casein solution at $100 \mathrm{mg} / \mathrm{mL}$ was prepared in PBS $(0.1 M, \mathrm{pH}$ 7.0) containing $\mathrm{Na}_{2} \mathrm{HPO}_{4}(0.577 \mathrm{~mol} / \mathrm{L})$ and $\mathrm{NaH}_{2} \mathrm{PO}_{4}$ $(0.423 \mathrm{~mol} / \mathrm{L})$. The protein solution $(5 \mu \mathrm{L})$ was applied dropwise onto clean chips and air-dried at room temperature. The chips with the attached protein were covered with $2.5 \%$ glutaraldehyde and kept overnight 
A

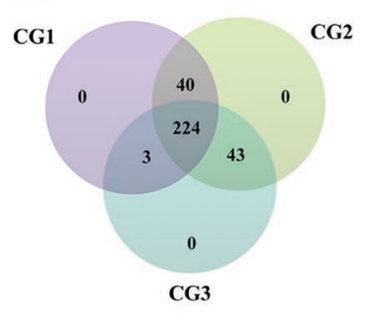

B

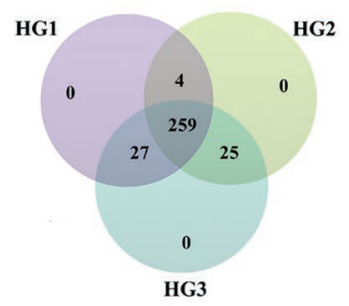

C

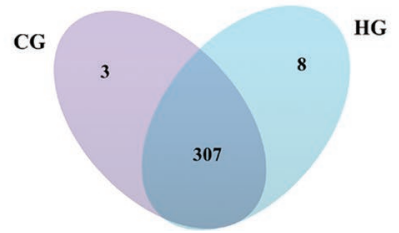

$\mathrm{D}$

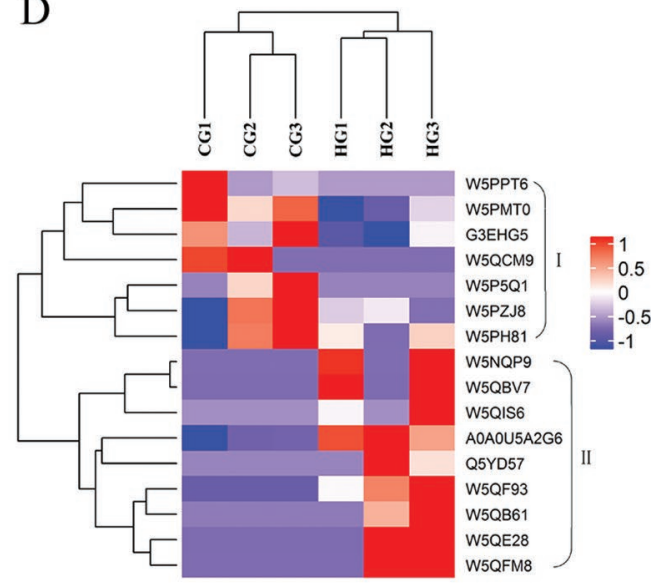

Figure 2. Venn diagram of quantified proteins in 3 biological duplicates of control group (CG; A) and homogenized group (HG; B) and the number of protein groups commonly present in goat milk from the CG and HG (C). Hierarchical clustering of differentially expressed proteins between the CG and HG (D).

at $4^{\circ} \mathrm{C}$, after which they were washed thrice with PBS. The samples were passed once through a graded ethanol series $(10,30,50,70,90,95$, and $100 \%)$ individually for 15 min each, transferred to a dryer, and incubated for $10 \mathrm{~min}$ at $37^{\circ} \mathrm{C}$ to prepare them for field emission scanning electron microscope analysis. The microstructure of WP and casein was analyzed with a SU8010 (Hitachi, Tokyo, Japan; http://www.hitachi.com/) field emission scanning electron microscope made in Japan.

\section{Fourier-Transform Infrared Measurements and Analysis}

The Fourier transform infrared (FTIR) spectra of the protein samples were recorded with an FTIR spectrometer (IRPrestige-21, Shimadzu, Kyoto, Japan) in the wavelength range 4,000 to $400 \mathrm{~cm}^{-1}$ with a resolution of $4 \mathrm{~cm}^{-1}$ using 10 scans. Spectral collection, peak identification, and basic infrared spectrogram analysis were performed using Omnic 8.2 (Nicolet, Thermo Fisher Scientific). The second derivative was calculated in the amide I region, 1,700 to $1,600 \mathrm{~cm}^{-1}$. Ten major peaks were resolved and fitted using Peakfit version 4.12 (Seasolve, San Jose, CA). A Gaussian function was used during the fitting routine for all peaks corresponding to $\alpha$-helix $\left(1,650-1,660 \mathrm{~cm}^{-1}\right), \beta$-sheet $(1,600-1,640$ $\mathrm{cm}^{-1}$ and 1,682-1,700 $\left.\mathrm{cm}^{-1}\right), \beta$-turn $\left(1,661-1,681 \mathrm{~cm}^{-1}\right)$, and random coil (1,641-1,650 $\mathrm{cm}^{-1}$; Long et al., 2015). The areas of all component bands assigned to a given secondary structural element were summed and divided by the total integrated area to yield the percentage of the element.

\section{RESULTS}

\section{Quantification and Hierarchical Clustering of Proteins}

We investigated the protein changes in goat milk after homogenization using label-free quantification. Venn diagrams showed 224 and 259 proteins common to the 3 biological replicates in the CG and HG samples as well as proteins uniquely expressed in goat milk in each group (Figure 2A and B). As seen in Figure 2C, 310 and 315 proteins were quantified in the CG and $\mathrm{HG}$, respectively, and 307 proteins were common between the 2 groups. Sixteen different proteins were also found between $\mathrm{CG}$ and $\mathrm{HG}$ by a $t$-test of the label-free quantification intensity data $(P<0.05$; Figure 2D). Cluster analysis of the different groups revealed that CG1, CG2, and CG3 clustered and that HG1, HG2, and HG3 were clustered, as depicted in Figure 2D. Furthermore, the cluster graph could be divided into clusters I and II (Figure 2D).

\section{GO Analysis of Identified Proteins}

Gene Ontology is a standardized system of gene function classification in which gene properties and products are described using the categories biological process (BP), molecular function (MF), and cellular component (CC). We determined the biological activity of proteins with significant differences $(P<0.05)$ based on MF, localized proteins based on $\mathrm{CC}$, and participating biological pathways based on BP (Figure $3)$. The main $\mathrm{BP}$ were involved in cellular process (12 
A

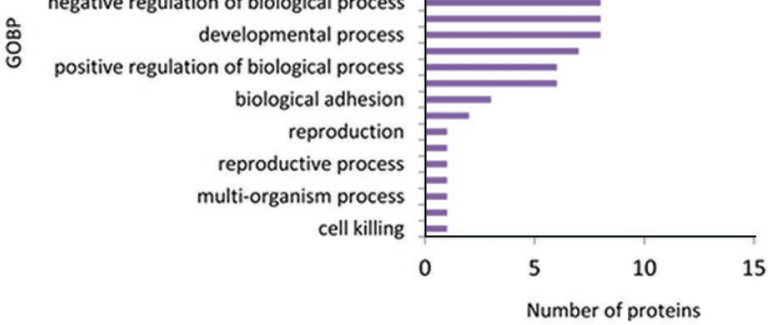

B
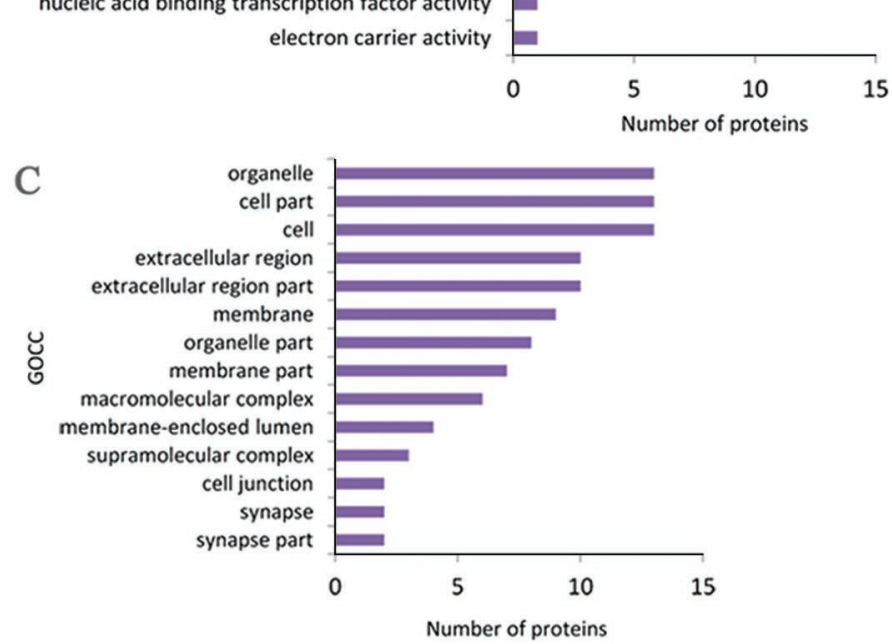

Figure 3. Gene Ontology (GO; http://www.geneontology.org/) analysis for biological processes (BP; A), molecular functions (MF; B), and cellular components $(\mathrm{CC} ; \mathrm{C})$ of identified proteins.

proteins) and single-organism process (11 proteins). Biological regulation (10 proteins) and regulation of biological process (10 proteins) also contributed to the $\mathrm{BP}$ designation. In addition, multicellular organismal process (9 proteins), localization (9 proteins), and response to stimulus (9 proteins) were well represented (Figure 3A).

The most obviously enriched MF for the identified proteins were related to binding (12 proteins) followed by catalytic activity ( 8 proteins; Figure $3 \mathrm{~B}$ ). The identified proteins were mainly described as from the organelle (13 proteins), cell (13 proteins), cell part (13 proteins), extracellular region part (10 proteins), and extracellular region (10 proteins). In addition, the membrane (9 proteins) was present in goat milk proteins (Figure 3C).

\section{Analysis of KEGG Pathways}

Homogenization affects protein structure and protein expression level (Figure 2D), which alters the metabolic pathways corresponding to the significantly different proteins $(P<0.05)$. Many metabolic pathways were tightly linked to glycolysis, as illustrated in Figure 4. These pathways included carbon fixation in photosynthetic organisms, fructose and mannose metabolism, carbon metabolism, biosynthesis of AA, purine metabolism, and caffeine metabolism.

\section{$\kappa-C N$ and $\beta-L G$ Proteins in Goat Milk from the $C G$ and $H G$}

To further validate the LC/MS results, the content of $\kappa-\mathrm{CN}$ and $\beta-\mathrm{LG}$ in the $\mathrm{CG}$ and $\mathrm{HG}$ were determined by ELISA. As Figure 5A shows, the standard curve coverage of $\kappa-\mathrm{CN}$ ranged from 6.25 to $200 \mu \mathrm{g} / \mathrm{mL}$. Figure $5 \mathrm{~B}$ shows that the standard curve of $\beta$-LG were 0 to 5 $\mathrm{ng} / \mathrm{mL}$. As expected, the variations of $\beta-\mathrm{LG}$ and $\kappa-\mathrm{CN}$ content in $\mathrm{CG}$ and $\mathrm{HG}$ as determined by ELISA were consistent with the LC/MS results (Figure $5 \mathrm{C}$ and D). In addition, Figure $5 \mathrm{C}$ and $\mathrm{D}$ revealed that $\kappa-\mathrm{CN}$ and $\beta$-LG levels in the HG were slightly higher than those in the CG, and these ELISA results also agreed with the LC/MS data. The consistent variation trends determined from the ELISA and LC/MS results indicate that the label-free technology used in this study was robust.

\section{Morphological Analysis of Goat Milk Protein}

The morphology of WP and casein from the CG and HG observed by field emission scanning electron microscopy is shown in Figure 6. The WP in CG (Figure 6A) and HG (Figure 6B) exhibits a regular spherical structure, but the WP in CG shows slight agglomeration. The distribution of WP particles in the homogenized goat milk was more uniform, the particle size was finer, the particle surface was smoother, and the arrangement was more regular, displaying no agglomeration. As depicted in Figure 6C and D, in fresh goat milk, casein particles were attached to each other to form micelles, and these micelles were connected to each other again to form a compact network structure. In the homogenized goat milk, the size distribution of the casein micelles was more uniform and the micelles were more spherical, the agglomeration phenomenon was substantially reduced, micelle size was reduced, and the surface morphology was more regular. 

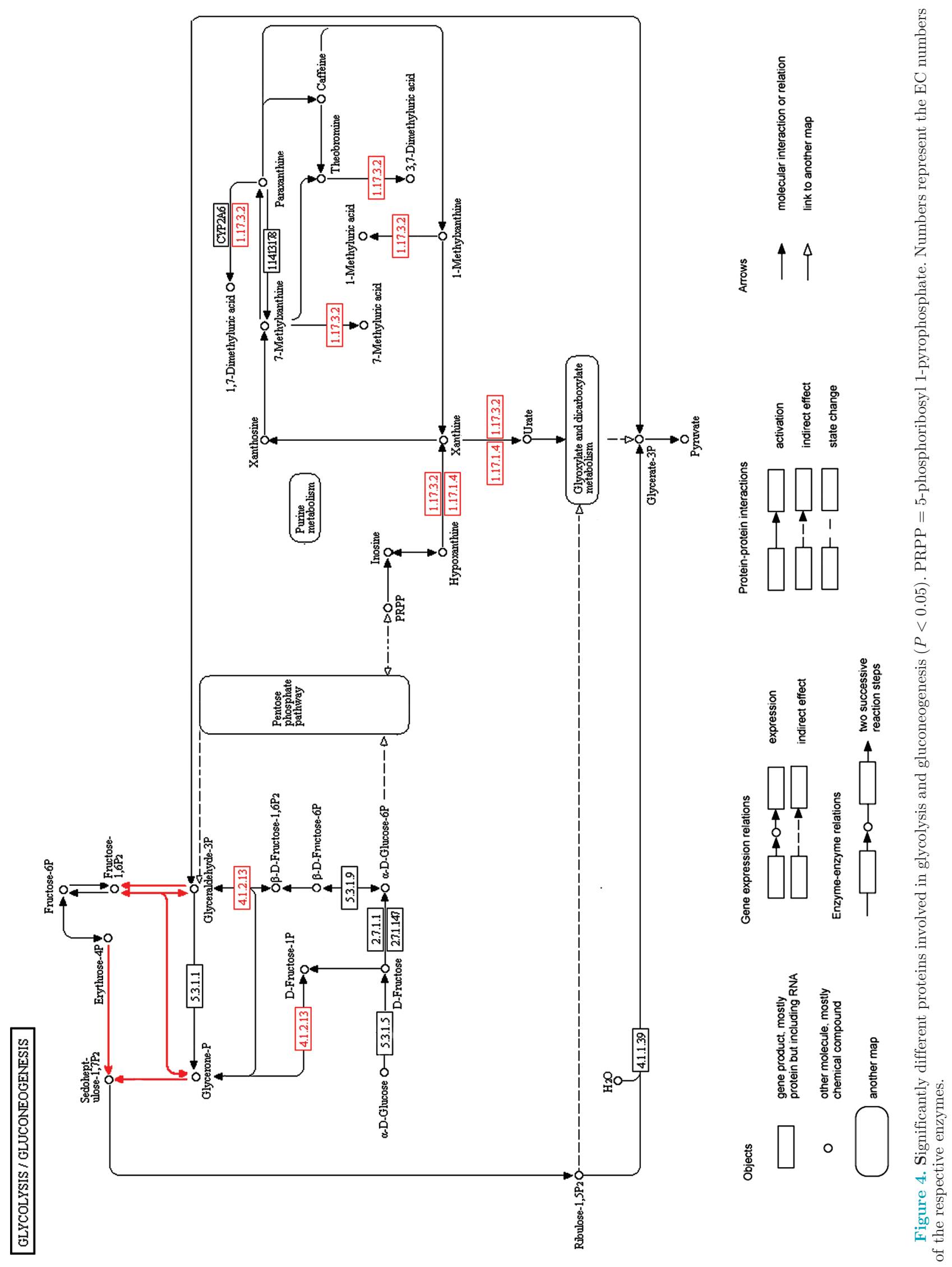


\section{FTIR Analysis of WP and Casein}

The apparent secondary structure of WP and casein from the CG and HG was analyzed using FTIR spectroscopic methods. Figure 7A, D, B, and E show FTIR spectra and the second derivative of the WP amide band from the CG and HG. The FTIR spectra in the amide I region $\left(1,700-1,600 \mathrm{~cm}^{-1}\right)$ is known for its sensitivity to protein secondary conformational change. The characteristic absorption peaks for raw milk and homogenized milk were 1,654.9 and 1,647.2 $\mathrm{cm}^{-1}$, respectively. As illustrated in Figure $7 \mathrm{C}$ and $\mathrm{F}$, the amide I region of the deconvoluted FTIR spectrum of WP from the CG was compared with that of WP from the HG. Figure $7 \mathrm{G}$ and $\mathrm{H}$ compare the secondary structural content of WP and casein from the CG with that of WP and casein from the HG. It was clear that there were significant differences in $\alpha$-helix, $\beta$-sheet, and random coil for WP between the CG and HG ( $P$ $<0.05$ ). However, for casein, there was no significant difference between the 2 groups $(P<0.05)$.

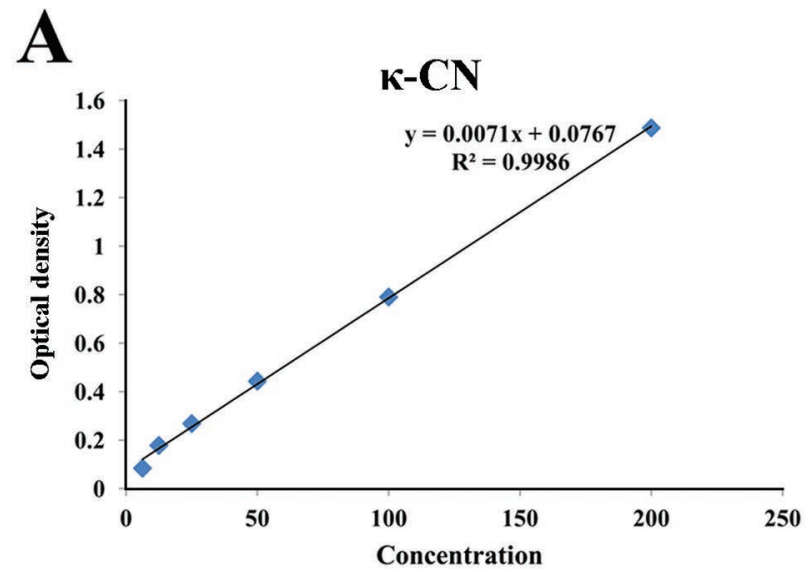

B

\section{Effect of Homogenization on Goat Milk Proteins}

We quantified 315 proteins in goat milk treated by homogenization (Figure 2C). Previously observed major proteins, such as $\beta$-LG (B2YKY6), $\kappa$-CN (A0A0U5A2G6), $\beta-\mathrm{CN}$ (Q5YD57), xanthine dehydrogenase (XDH; W5PMT0), and aldolase (ALDO; W5NQP9), were all identified at high abundance in our study, which indicates the robustness of the methodology (Figure 2D). In previous investigations, 423 proteins were identified and XDH was significantly different in abundance between the colostrum and mature goat milk (Lu et al., 2016). In addition, 53 unique carbonylated peptides (37 carbonylation sites, 15 proteins) were identified in raw milk and differently processed milk products (Milkovska-Stamenova et al., 2017). We found 16 proteins to be significantly different between fresh milk and homogenized milk $(P<0.05)$. Figure 2D clearly shows that protein expression in cluster I of the CG
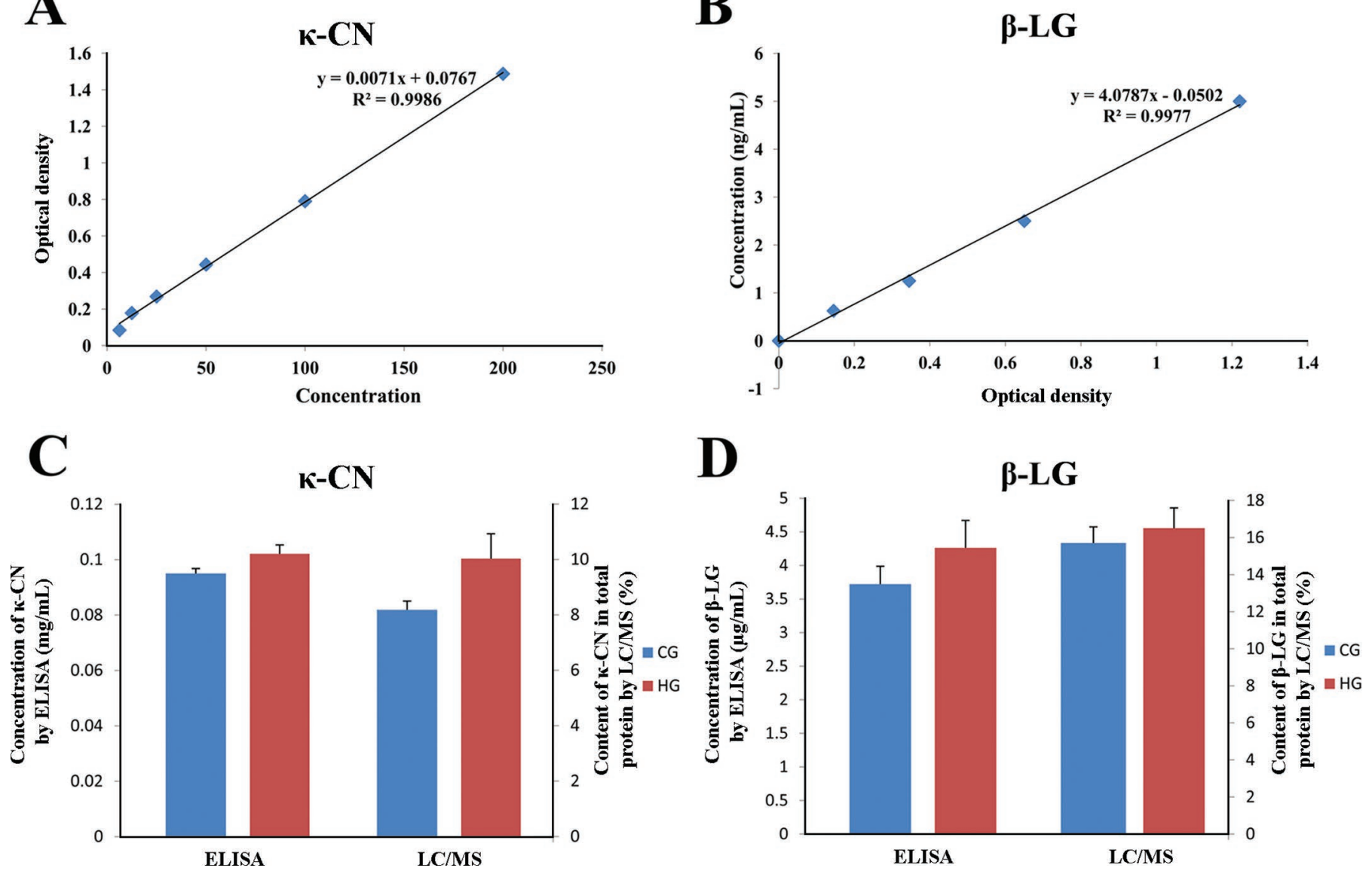

Figure 5. Standard curves for $\kappa-\mathrm{CN}(\mathrm{A})$ and $\beta$-LG (B) by ELISA and comparison of LC/MS and ELISA results for $\kappa-\mathrm{CN}$ (C) and $\beta$-LG (D). $\mathrm{CG}=$ control group; $\mathrm{HG}=$ homogenized group. Error bars represent SD. 
was higher than that of the HG; in contrast, the protein expression in cluster II showed the opposite trend. The clustering results demonstrated protein degradation and denaturation in goat milk during homogenization and the formation of new products. In the homogenized groups, XDH showed lower expression and ALDO was abundant protein (Figure 2D). This tendency indicated that homogenization destroyed the original structure of fat globules. Analysis of these proteins would increase our understanding and knowledge of goat milk proteins after homogenization treatment.

Several studies have focused on the effects of homogenization on fat globule microstructure in cow milk used confocal laser scanning microscopy (Yao et al., 2015).
Cadesky et al. (2017) observed structural changes induced by high-pressure processing in casein micellar and milk protein concentrates. Previous research has shown that homogenization reduces milk fat globule size and decreases globule size range, resulting in a newly formed membrane (Qi et al., 2015). We focused on changes in WP and casein micelles (Figure 6), and morphology results showed that homogenization reduced WP size and increased protein distribution uniformity, with no obvious agglomeration phenomena observed (Figure 6A and $\mathrm{B}$ ). Figure $7 \mathrm{G}$ shows that the $\alpha$-helix content in the $\mathrm{HG}$ was much higher than that in the CG, although the percentage of random coil in the HG was significantly reduced compared with that in the CG $(P<0.05)$,
A

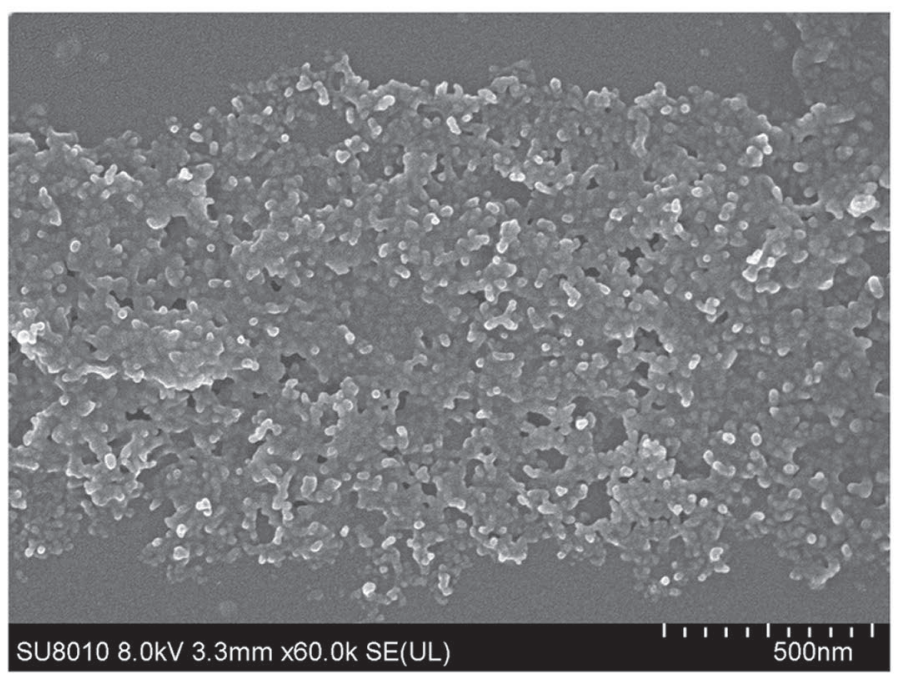

$\mathrm{C}$

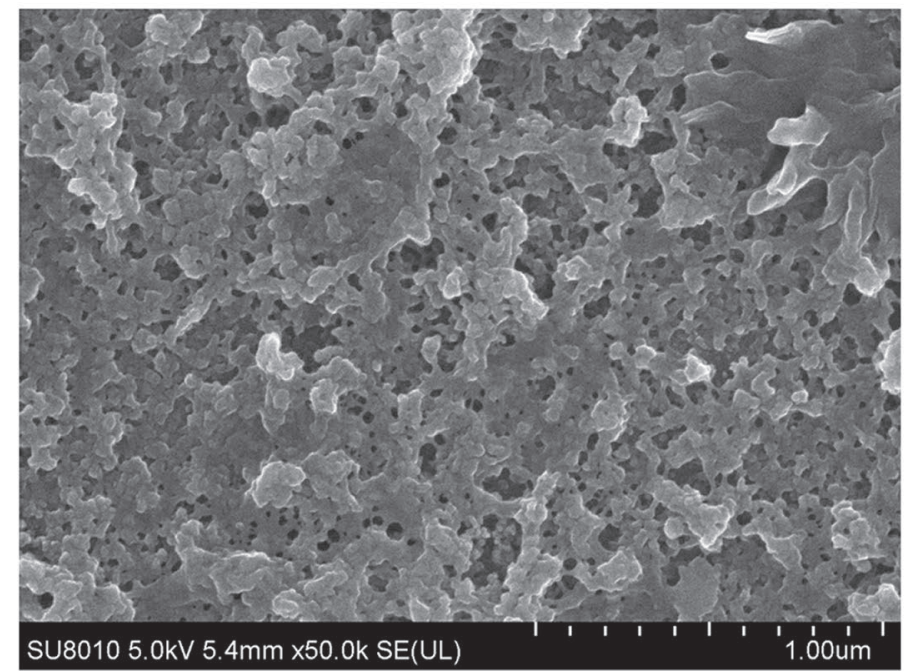

B

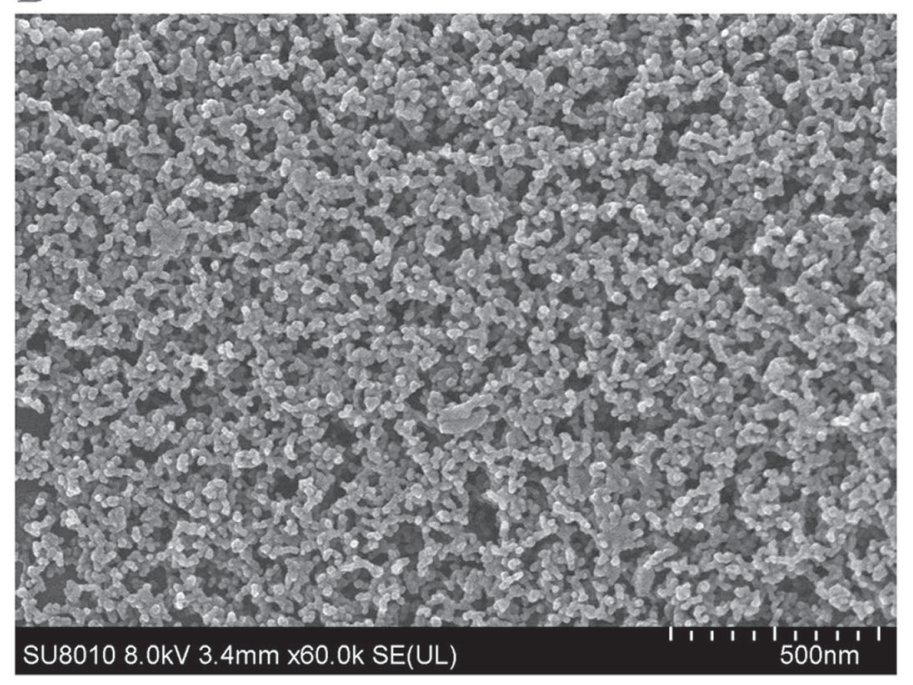

D

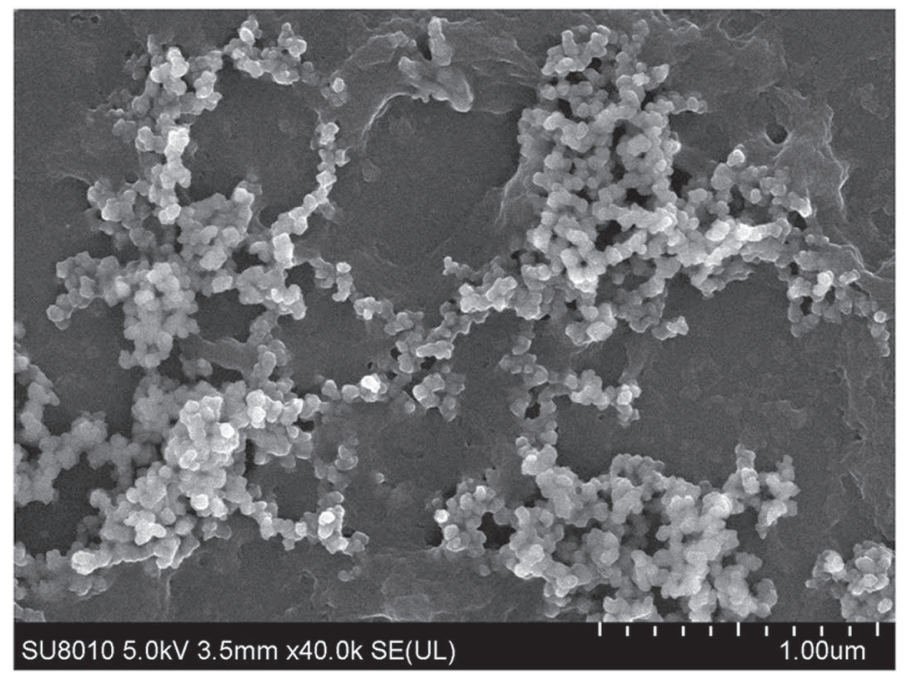

Figure 6. Field emission scanning electron microscope images of whey protein and casein from the control group and homogenized group. A

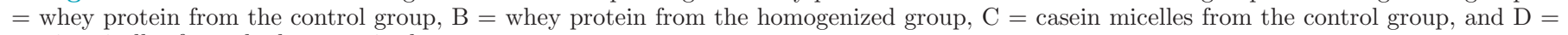
casein micelles from the homogenized group. 
which further indicates that homogenization stabilized WP. By changing milk protein granule size with homogenization treatment, the physicochemical properties of proteins, such as color, viscosity, $\mathrm{pH}$, and acidity, were also affected (Amador-Espejo et al., 2014). Ultimately, homogenization reduced the heat coagulation time and the thermal stability of milk (Meena et al., 2018). The effects of heat coagulation time on microstructure and rheological properties, including solubility, heat stability, water binding, dispersibility, foaming, and emulsifying of hazelnut milks, have been investigated (Gul et al., 2017).
Homogenization disrupts fat droplets and causes the globule membrane to rearrange, resulting in increased interactions between WP, casein, and fat globules (Qi et al., 2015). Figure 2D shows significant differences in the expression of XDH (W5PMT0) and asparaginase like 1 (ASRGL1; W5PZJ8) between the CG and HG $(P<0.05)$. The $\mathrm{XDH}$ was the most abundant protein in goat milk fat globule membrane (Lu et al., 2016). In addition, XDH (part of the MFGMP) was partially dissociated on the milk fat globule membrane surface (Fauquant et al., 2010). One possible reason for the decrease in $\mathrm{XDH}$ and ASRGL1 is the destruction of
A
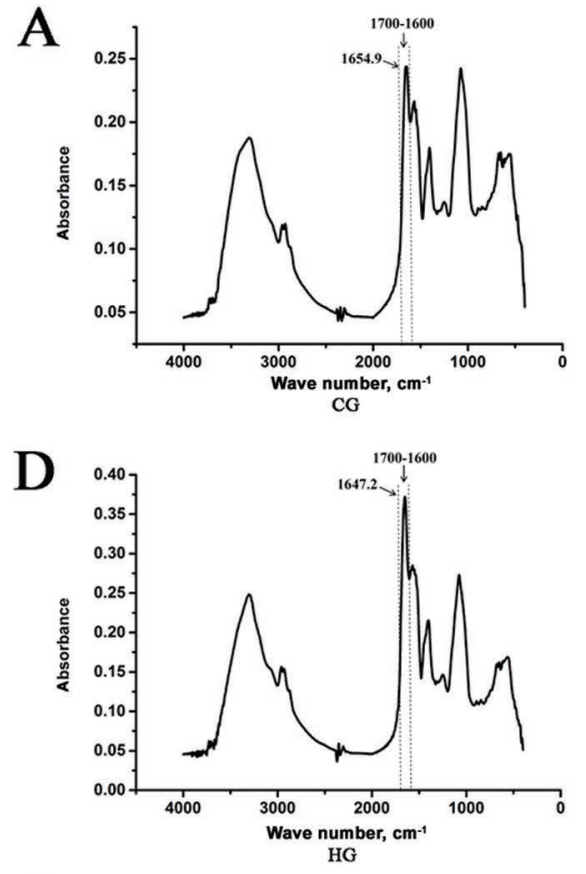

G

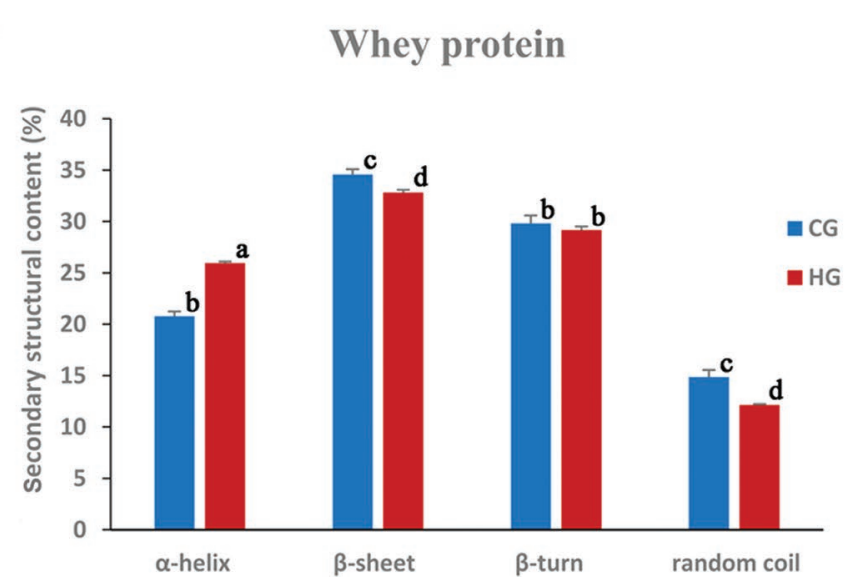

B

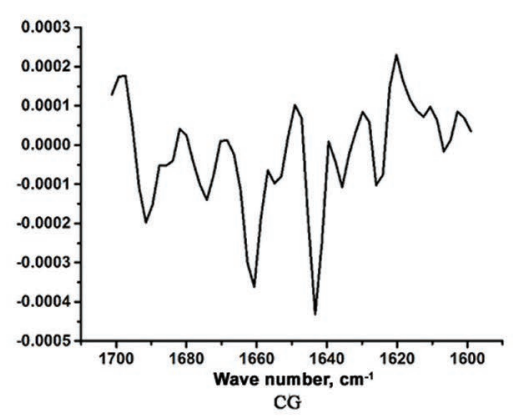

E

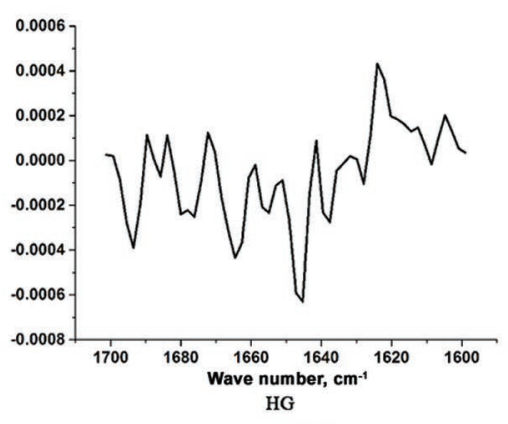

$\mathrm{H}$

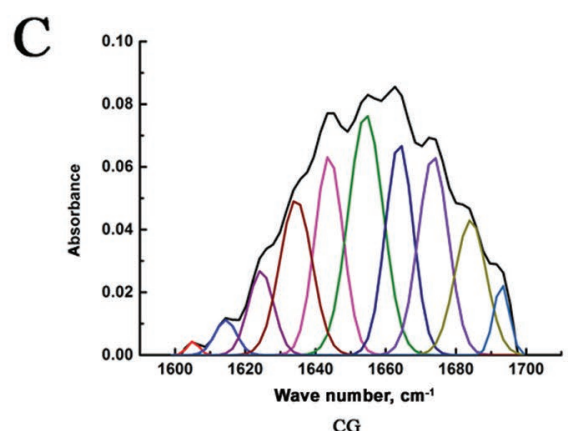

F

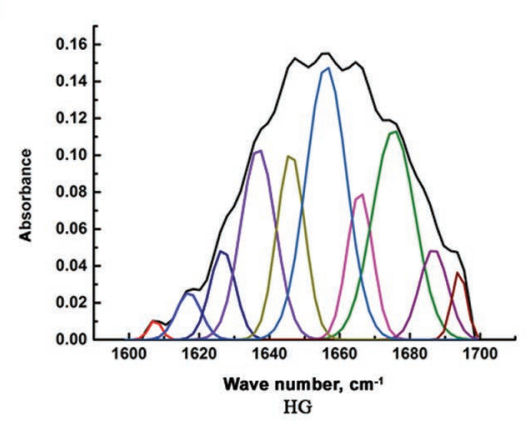

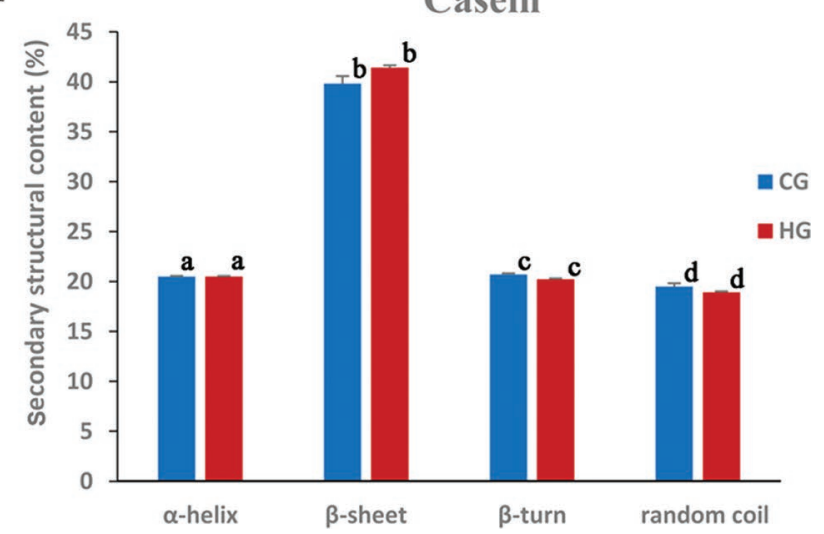

Figure 7. Fourier transform infrared spectra of the control group (CG; A) and homogenized group (HG; D); the second derivatives of amide I of the CG (B) and HG (E); Gaussian curve fitting of amide I of the CG (C) and HG (F); and comparison of secondary structural content of WP $(\mathrm{G})$ and $\mathrm{CN}(\mathrm{H})$ between the CG and HG. Different letters $(\mathrm{a}-\mathrm{d})$ indicate significant differences $(P<0.05)$ between the CG and HG. Error bars represent SD. 
fat globulin protein during homogenization. When the milk samples were homogenized, $\mathrm{XDH}$ was removed from the fresh goat milk. In addition, the shear force and heat might destroy enzyme activity, resulting in decreased ASRGL1 levels.

Meena et al. (2018) suggested that homogenization disrupted casein aggregates due to shearing and dispersed them uniformly. The treatment enhanced the net negative charge and electrostatic repulsive forces between the casein micelles, preventing casein aggregation. As seen in Figure $6 \mathrm{C}$ and $\mathrm{D}$, the original casein micelle structure was destroyed. Additionally, particle size distribution was relatively uniform, the agglomeration phenomenon was substantially reduced, and aggregation was decreased (Figure $6 \mathrm{C}$ and $\mathrm{D}$ ). We noted a possible increase in $\kappa-\mathrm{CN}$ dissociation, which was validated by data showing that $\kappa-\mathrm{CN}$ levels in the HG were higher than those in the CG by ELISA and LC/MS (Figure 5). As expected, the content of $\kappa$-CN and $\beta-\mathrm{CN}$ in cluster I of the HG were also higher than those in the CG (Figure 2D). Due to homogenization, original internal $\beta-\mathrm{CN}$ was liberated from the destroyed casein micelles and was therefore significantly higher in the HG than in the CG $(P<0.05$; Figure 2D). This result validated earlier reports indicating that the $\alpha_{\mathrm{S} 1}-\mathrm{CN}$ percentage does not change, although the $\kappa-\mathrm{CN}$ percentage increased and the dissociation of $\beta$-LG slightly increased (Cano-Ruiz and Richter, 1997). This was due to $\mathrm{k}-\mathrm{CN}$ dissociation from the fat globule surface, regardless of changes to the casein micelle microstructure. However, Figure $7 \mathrm{H}$ indicated that homogenization did not significantly change casein secondary structure $(P$ $<0.05)$. For CG, the fat globules were simply trapped within the gel structure formed by the casein micelles, whereas for HG the fat globules were coated with caseins and actively are incorporated into the gel matrix (Ye et al., 2017). The globules themselves help form the matrix as the clot forms from smaller fat globules in the homogenized milk (Cano-Ruiz and Richter, 1997). Thus, homogenized goat milk is more conducive to clot formation, and this change has a large influence on yogurt and cheese processing.

Figure 5D shows that the $\beta$-LG content in the HG was higher than that in the CG. In ultra-high-pressure homogenization-treated milk, small amounts of $\beta$-LG might denature, which is likely due to the increase in mechanical forces and temperature (Qi et al., 2015) resulting from the transformation between sulfhydryl groups (-SH) and disulfide bonds (-S-S-; Hernández and Harte, 2008). The secondary structure of WP changed and ultimately increased in stability (Qi et al., 2015). Sulfhydryl groups and disulfide bonds are important for protein structure, protein-protein interaction, and function. Unfortunately, the destructive power was much less than heat damage (Pereda et al., 2009 ), which is why we observed no significant change in $\beta$-LG solely due to homogenization, although its level increased slightly $(P<0.05)$. Qi et al. (2015) showed that conventional homogenization treatment does not appear to affect the levels of free sulfhydryl in soluble WP (e.g., $\beta-L G, \alpha-L A, \operatorname{IgG}$ ) in whole milk compared with those in their skimmed counterparts.

\section{Proteins Primarily Involved in GO}

Gene Ontology results suggested that cellular processes had the largest proportion in $\mathrm{BP}$, and binding was the most important in MF (Figure 3). At the same time, organelle, cell, and cell part were dominant in CC (Figure 3). In a recent paper, MFGMP mainly originated from the plasma membrane (59 proteins), cytosol (32 proteins), and cytoplasmic vesicle (31 proteins; Lu et al., 2016). According to previous research, localization for the major classes of WP from sheep is extracellular, followed by membrane and cytoplasmic (Anagnostopoulos et al., 2016). Yang et al. (2017) found that proteins combined with a partner molecule or other proteins to form protein complexes that expand protein function. For example, specific binding to antigens leads to complement activation and promote phagocytosis, WP binding to proteins and ions, and then association with hydrolase and enzyme inhibitor activity.

Based on GO functional annotations in other research, quantified bovine milk WP are related to cellular processes, intracellular organelles, and molecular functions (Yang et al., 2017). Lu et al. (2016) noted that MFGMP were involved in complement and coagulation cascades, whereas adherens junction and galactose metabolism were only observed in colostrum. We focused on the effect of homogenization treatment on ALDO. In our study, ALDO in BP included cellular component organization or biogenesis, developmental process, cellular process, single-organism process, metabolic process, multicellular organismal process, regulation of biological process, biological adhesion, localization, and biological regulation. The MF for ALDO included binding and catalytic activity, as shown in Figure 3; ALDO was also involved in all CC in Figure 3 except for membrane part, synapse part, and synapse. On the basis of these findings, ALDO is involved in glycolysis and gluconeogenesis metabolism.

\section{KEGG Pathway Analysis}

Ye et al. (2017) studied the effect of homogenization and heat treatment on the behavior of protein and fat globules during the gastric digestion of milk. 
After homogenization, milk fat is easily digested and absorbed because the diameter of the milk fat globule is reduced. Michalski and Januel (2006) suggested that changes in homogenized milk could improve milk digestibility and enhance protein bioavailability. The fat and lactose molecules of goat milk shrank during homogenization, and smaller lactose particles are more conducive to digestion and absorption (Korpela et al., 2005). In the human small intestine, lactose is hydrolyzed by lactase into glucose and galactose and then absorbed. Hexokinase, phosphofructokinase-1, and pyruvate kinase are key enzymes in glycolysis, especially phosphofructokinase-1 (Yang et al., 2011). In our study, we noted a significant difference in ALDO expression after homogenization $(P<0.05)$, and ALDO also had an effect on glycolysis (Figure 4). This could be because homogenization causes changes in protein expression, which in turn affects glycolysis and gluconeogenesis.

In general, ALDO showed significant changes in expression between the 2 groups $(P<0.05)$, with the HG showing higher levels (Figure 2D). Aldolase promotes conversion of glyceraldehyde-3P into sedoheptulose-1,7bisphosphate through carbon fixation in photosynthetic organisms (Figure 4), which ultimately improves glycerate-3P production. During fructose and mannose metabolism, ALDO converts D-fructose to generate Dfructose-1P and glycerone-P (Figure 4); ALDO also converts fructose-6P to glycerone-P in carbon metabolism, which can be converted to glyceraldehyde-3P (Figure 4). Via glycolysis and gluconeogenesis, ALDO converts $\alpha / \beta$-D-glucose generated from $\beta$-D-fructose- $1,6 \mathrm{P}_{2}$ to glyceraldehyde-3P (Figure 4). Finally, ALDO converts glyceraldehyde-3P to glycerate-3P during glycolysis metabolism (Figure 4). Therefore, the presence of ALDO during homogenization promotes the conversion of glucose sugar to pyruvate. In the role of metabolism, pyruvate mainly (1) reduces to lactic acid, providing energy, and (2) goes into mitochondria to form acetyl CoA for the citrate cycle (Nelson and Cox, 2005).

In addition, KEGG analysis indicated that xanthinetransformed glyoxylate also participates in glyoxylate and dicarboxylate metabolism. The XDH expression was significantly higher in the CG than the HG $(P<$ 0.05; Figure 2D). From caffeine metabolism, we found that the HG accumulates more xanthine (Figure 4). In addition, $\mathrm{XDH}$ has low levels in the HG, so xanthine was more likely to be converted to glycine than urate in purine metabolism (Figure 4). This conversion reduces the pressure on glycolysis and gluconeogenesis. Based on the metabolic pathways altered by proteins that are significantly different after homogenization $(P<0.05)$, homogenization improves glycolysis and gluconeogenesis in goat milk; this may promote the production of pyruvic acid. Pyruvate can realize the mutual transformation of sugar, fat, and AA in vivo through acetyl-CoA and tricarboxylic acid cycle (Nelson and Cox, 2005). Moreover, changes in external conditions (such as pressure or temperature) produce different effects in goat milk (Pinho et al., 2011b). Previous studies found that ultra-high pressure homogenization destroys microorganisms mechanically and with sudden pressure drops (Dumay et al., 2013). Ultra-high-pressure homogenization was also shown to preserve vitamin $\mathrm{C}$ and riboflavin better than thermal milk treatment (Sharabi et al., 2018). This technology has been used to improve rheological characteristics, physical properties, and milk stability as well as the polysaccharide and protein characteristics (D'Incecco et al., 2018). Homogenization and heat treatment can simultaneously improve the production process of dairy products (Li et al., 2018b).

\section{CONCLUSIONS}

By using microstructure information, we found that the goat milk protein became smaller and more evenly distributed, increasing the regular arrangement of the secondary structures, during the homogenization process. Proteomics analysis verified that the expression of XDH and ASRGL1 was higher in CG than in HG, whereas the opposite was observed for ALDO, $\kappa-\mathrm{CN}$, and $\beta-C N$. Significant changes were found in the homogenized goat milk proteomics, which were related to glycolysis and gluconeogenesis. Based on this experimental study, future research will be dedicated to identifying the proteins involved in the deposits formed under homogenization of goat milk. It is important to study the effects of various homogeneous conditions of proteins through proteomics and then observe the changes in metabolism rather than just performing sensory evaluation in goat milk.

\section{ACKNOWLEDGMENTS}

This work was supported by the National Natural Science Foundation of China (31501501), Natural Science Foundation of Shandong Province (ZR2014JL019). Technical support by Shanghai Applied Protein Technology (Shanghai, China) is gratefully acknowledged.

\section{REFERENCES}

Amador-Espejo, G. G., A. Suàrez-Berencia, B. Juan, M. E. Bárcenas, and A. J. Trujillo. 2014. Effect of moderate inlet temperatures in ultra-high-pressure homogenization treatments on physicochemical and sensory characteristics of milk. J. Dairy Sci. 97:659-671.

Anagnostopoulos, A. K., A. I. Katsafadou, V. Pierros, E. Kontopodis, G. C. Fthenakis, G. Arsenos, S. C. Karkabounas, A. Tzora, I. Skoufos, and G. T. Tsangaris. 2016. Milk of Greek sheep and goat 
breeds; characterization by means of proteomics. J. Proteomics 147:76-84.

Brick, T., M. Ege, S. Boeren, A. Böck, E. M. Von, J. Vervoort, and K. Hettinga. 2017. Effect of processing intensity on immunologically active bovine milk serum proteins. Nutrients 9:963.

Cadesky, L., M. Walkling-Ribeiro, K. T. Kriner, M. V. Karwe, and C. I. Moraru. 2017. Structural changes induced by high-pressure processing in micellar casein and milk protein concentrates. J. Dairy Sci. 100:7055.

Cano-Ruiz, M. E., and R. L. Richter. 1997. Effect of homogenization pressure on the milk fat globule membrane proteins. J. Dairy Sci. 80:2732-2739.

Cox, J., M. Y. Hein, C. A. Luber, I. Paron, N. Nagaraj, and M. Mann 2014. Accurate proteome-wide label-free quantification by delayed normalization and maximal peptide ratio extraction, termed MaxLFQ. Mol. Cell. Proteomics 13:2513.

D'Incecco, P., V. Rosi, G. Cabassi, J. A. Hogenboom, and L. Pellegrino. 2018. Microfiltration and ultra-high-pressure homogenization for extending the shelf-storage stability of UHT milk. Food Res. Int. 107:477-485.

Chen, D., X. Zhao, X. Li, J. Wang, and C. Wang. 2018. Milk compositional changes of Laoshan goat milk from partum up to 261 days postpartum. Anim. Sci. J. 89:1355-1363.

Dumay, E., D. Chevalier-Lucia, L. Picart-Palmade, A. Benzaria, A. Gràcia-Julià, and C. Blayo. 2013. Technological aspects and potential applications of (ultra) high-pressure homogenisation. Trends Food Sci. Technol. 31:13-26.

Fauquant, C., V. Briard, N. Leconte, and M. C. Michalski. 2010. Differently sized native milk fat globules separated by microfiltration: Fatty acid composition of the milk fat globule membrane and triglyceride core. Eur. J. Lipid Sci. Technol. 107:80-86.

Götz, S., J. M. Garcia-Gomez, J. Terol, T. D. Williams, S. H. Nagaraj, M. J. Nueda, M. Robles, M. Talon, J. Dopazo, and A. Conesa. 2008. High-throughput functional annotation and data mining with the Blast2GO suite. Nucl. Acids Res. 36:3420-3435.

Gul, O., F. T. Saricaoglu, M. Mortas, I. Atalar, and F. Yazici. 2017. Effect of high pressure homogenization (HPH) on microstructure and rheological properties of hazelnut milk. Innov. Food Sci. Emerg. Technol. 41:411-420.

Hernández, A., and F. M. Harte. 2008. Manufacture of acid gels from skim milk using high-pressure homogenization. J. Dairy Sci. 91:3761-3767.

Huppertz, T. 2011. Homogenization of milk: High-pressure homogenizers. Pages 755-760 in Encyclopedia of Dairy Sciences, Vol. 10. NIZO Food Research, Ede, the Netherlands.

Korpela, R., L. Paajanen, and T. Tuure. 2005. Homogenization of milk has no effect on the gastrointestinal symptoms of lactose intolerant subjects. Milchwissenschaft 60:3-6.

Le, T. T., H. C. Deeth, and L. B. Larsen. 2017. Proteomics of major bovine milk proteins: Novel insights. Int. Dairy J. 67:2-15.

Li, Y., D. Chen, J. Li, X.-X. Zhang, C.-F. Wang, and J.-M. Wang. 2018a. Changes in superoxide dismutase activity postpartum from Laoshan goat milk and factors influencing its stability during processing. Ital. J. Anim. Sci. 17:835-844.

Li, Y., H. S. Joyner Melito, B. G. Carter, and M. A. Drake. 2018b. Effects of fat content, pasteurization method, homogenization pressure, and storage time on the mechanical and sensory properties of bovine milk. J. Dairy Sci. 101:2941-2955.

Liang, Y., L. Matia-Merino, G. Gillies, H. Patel, A. Ye, and M. Golding. 2017. The heat stability of milk protein-stabilized oil-in-water emulsions: A review. Curr. Opin. Colloid Interface Sci. 28:63-73.

Long, G., Y. Ji, H. Pan, Z. Sun, Y. Li, and G. Qin. 2015. Characterization of thermal denaturation structure and morphology of soy glycinin by FTIR and SEM. Int. J. Food Prop. 18:763-774.

Lu, J., L. Liu, X. Pang, S. Zhang, Z. Jia, C. Ma, L. Zhao, and J. Lv. 2016. Comparative proteomics of milk fat globule membrane in goat colostrum and mature milk. Food Chem. 209:10-16.
Lu, J., X. Wang, W. Zhang, L. Liu, X. Pang, S. Zhang, and J. Lv 2016. Comparative proteomics of milk fat globule membrane in different species reveals variations in lactation and nutrition. Food Chem. 196:665-672.

Meena, G. S., A. K. Singh, V. K. Gupta, S. Borad, S. Arora, and S. K. Tomar. 2018. Effect of $\mathrm{pH}$ adjustment, homogenization and diafiltration on physicochemical, reconstitution, functional and rheological properties of medium protein milk protein concentrates (MPC70). J. Food Sci. Technol. 55:1376-1386.

Michalski, M. C., and C. Januel. 2006. Does homogenization affect the human health properties of cow's milk? Trends Food Sci. Technol. $17: 423-437$.

Milkovska-Stamenova, S., R. Mnatsakanyan, and R. Hoffmann. 2017. Protein carbonylation sites in bovine raw milk and processed milk products. Food Chem. 229:417.

Moriya, Y., M. Itoh, S. Okuda, A. Yoshizawa, and M. Kanehisa. 2007. KAAS: An automatic genome annotation and pathway reconstruction server. Nucl. Acids Res. 35(Suppl. 2):W182-W185.

Nelson, D. L., and M. M. Cox. 2005. Lehninger Principles of Biochemistry. W. H. Freeman, London, UK.

Pereda, J., V. Ferragut, J. M. Quevedo, B. Guamis, and A. J. Trujillo. 2009. Heat damage evaluation in ultra-high pressure homogenized milk. Food Hydrocoll. 23:1974-1979.

Pereda, J., D. P. Jaramillo, J. M. Quevedo, V. Ferragut, B. Guamis, and A. J. Trujillo. 2008. Characterization of volatile compounds in ultra-high-pressure homogenized milk. Int. Dairy J. 18:826-834.

Pinho, C. R. G., M. A. Franchi, A. A. L. Tribst, and M. Cristianini. 2011a. Effect of ultra high pressure homogenization on alkaline phosphatase and lactoperoxidase activity in raw skim milk. Procedia Food Sci. 1:874-878.

Pinho, C. R. G., M. A. Franchi, A. A. L. Tribst, and M. Cristianinia. 2011b. Effect of high pressure homogenization process on Bacillus stearothermophilus and Clostridium sporogenes spores in skim milk. Procedia Food Sci. 1:869-873.

Qi, P. X., D. Ren, Y. Xiao, and P. M. Tomasula. 2015. Effect of homogenization and pasteurization on the structure and stability of whey protein in milk. J. Dairy Sci. 98:2884-2897.

Sharabi, S., Z. Okun, and A. Shpigelman. 2018. Changes in the shelf life stability of riboflavin, vitamin $\mathrm{C}$ and antioxidant properties of milk after (ultra) high pressure homogenization: Direct and indirect effects. Innov. Food Sci. Emerg. Technol. 47:161-169.

Wisniewski, J. R., A. Zougman, N. Nagaraj, and M. Mann. 2009. Universal sample preparation method for proteome analysis. Nat. Methods 6:359-362.

Yang, M., X. Cao, R. Wu, B. Liu, W. Ye, X. Yue, and J. Wu. 2017. Comparative proteomic exploration of whey proteins in human and bovine colostrum and mature milk using iTRAQ-coupled LCMS/MS. Int. J. Food Sci. Nutr. 68:671-681.

Yang, J., D. An, and P. Zhang. 2011. Expression profiling of cassava storage roots reveals an active process of glycolysis/gluconeogenesis. J. Integr. Plant Biol. 53:193-211.

Yao, Y., G. Zhao, X. Zou, L. Huang, and X. Wang. 2015. Microstructural and lipid composition changes in milk fat globules during milk powder manufacture. RSC Advances 5:62638-62646.

Ye, A., J. Cui, D. Dalgleish, and H. Singh. 2017. Effect of homogenization and heat treatment on the behavior of protein and fat globules during gastric digestion of milk. J. Dairy Sci. 100:36-47.

Zhu, Y., J. Wang, and C. Wang. 2018. Research on the preparation, uniformity and stability of mixed standard substance for rapid detection of goat milk composition. Anim. Sci. J. 89:794-801.

Zhu, Y., H. Xu, H. Chen, J. Xie, M. Shi, B. Shen, X. Deng, C. Liu, X. Zhan, and C. Peng. 2014. Proteomic analysis of solid pseudopapillary tumor of the pancreas reveals dysfunction of the endoplasmic reticulum protein processing pathway. Mol. Cell. Proteomics $13: 2593-2603$ 